\title{
Nivel de calidad de vida laboral en docentes de facultades de educación de universidades públicas de la macro región centro
}

\author{
Metodio Cirilo Quispe Aclari \\ Universidad Nacional del Centro del Perú•mcquispe@uncp.edu.pe \\ Esteban Medrano Reynoso \\ Universidad Nacional del Centro del Perú•emedrano@uncp.edu.pe \\ https://orcid.org/0000-0002-9512-6305 \\ Elvis Vidal Ramírez Calzada \\ Universidad Nacional del Centro del Perú•eramirez@uncp.edu.pe \\ https://orcid.org/0000-0001-7649-0358 \\ Joseph Jhoel Virrueta Antezano \\ e_2017200853g@uncp.edu.pe \\ Helen Elvi Quispe Cerrón \\ hequispe@uncp.edu.pe
}

\section{Resumen}

La investigación tuvo como propósito el estudio de los niveles de calidad de vida laboral de los docentes de facultades de Educación de las universidades estatales de la macro región centro, cuyos resultados se obtuvieron mediante la aplicación de un cuestionario a un grupo muestral. La investigación corresponde al tipo básica, nivel descriptivo, donde se empleó el método científico como método general en todo el proceso de la investigación. La población y la muestra estuvo constituido por los docentes de ambos géneros de la Universidad Nacional del Centro del Perú, Universidad Nacional de Huancavelica y la Universidad "Hermilio Valdizán" en su condición de contratado y nombrado y por categorías. Para el procesamiento de datos, resultado de la aplicación del cuestionario de nivel de calidad de vida laboral, la misma que se aplicó utilizando la herramienta formulario de Google Workspace, para el análisis se empleó la estadística descriptiva y la estadística inferencial y con ayuda del programa SPSS que permitió la interpretación de los resultados, a partir de ello se ha concluido, no existen diferencias significativas en los docentes de las universidades de la macro región centro en relación a la calidad de vida laboral, ya que la chi cuadrada calculada fue menor que chi cuadrada teórica $(8,34<11,143)$, obteniendo un nivel de significancia de 0,05 .

Palabras clave: Calidad de vida, laboral, docentes, nivel de vida.

\begin{abstract}
The purpose of the research was to study the levels of quality of working life of teachers of faculties of education of public universities in the central macro region, the results of which were obtained by applying a questionnaire to a sample group. The research corresponds to the basic type, descriptive level, where the scientific method was used as a general method throughout the research process. The population and the sample consisted of teachers of both genders from the National University of the Center of Peru, the National University of Huancavelica and the "Hermilio Valdizan" University in their condition of contracted and appointed and by categories. For data processing, the result of the application of the questionnaire on the level of quality of working life, the same that was applied using the Google Workspace form tool, for the analysis descriptive statistics and inferential statistics were used and with the help of the program SPSS that allowed the interpretation of the results. According to the data obtained, it has been concluded that there are no significant differences in the levels of quality of working life in teachers of the faculties of education of the public universities of the central macro-region, since the calculated chi-square was less than the theoretical chi-square $(8.34<11.143)$, with a significance level of 0.05 .
\end{abstract}

Keywords: Quality of life, work, teachers, standard of living. 


\section{Introducción}

El fenómeno del COVID-19, es una pandemia que ha trastocado diferentes aspectos de la vida humana, como el medio ambiente, la salubridad, la economía, la producción, educación, la conectividad virtual, entre otros aspectos. El docente universitario no es ajeno a ello, pues ha cambiado radicalmente la forma de desarrollo de la gestión pedagógica y gestión administrativa, la interrelación con los estudiantes, colegas, autoridades, etc. sobre todo la metodología del proceso enseñanza-aprendizaje a través de las redes sociales y por algunas plataformas virtuales. Para muchos seguramente constituye dificultades enormes involucrarse en esta nueva forma de interactuar, por las dificultades en la conectividad, sin embargo, se viene superando seguramente por la predisposición y la perseverancia, pensando en un futuro mejor para los estudiantes. El logro de las competencias, las capacidades y los desempeños son los propósitos en la formación profesional de los estudiantes que les permita en el futuro desempeñarse con eficiencia, creatividad y otras habilidades blandas propios de un profesional líder.

Por las experiencias que se viene plasmando, todos exigen el logro de las competencias, la calidad educativa, dominio de la tecnología, la conectividad, entre otras exigencias de carácter académico y administrativa, desde la Superintendencia Nacional de Educación Superior Universitaria (SUNEDU), autoridades universitarias y las facultades, pero nunca se pronuncian en la calidad de vida de los docentes que realizan enormes esfuerzos por cumplir con las exigencias impuestas, es más, vienen laborando más de las 8 horas, incluido los sábados y domingos, aduciendo que se deben cumplir por vocación y el servicio de calidad a los usuarios. Por ende, se plantea el problema general: ¿Existen diferencias en los niveles de calidad de vida laboral en los docentes de las facultades de educación de las universidades públicas de la macro región centro?

Así mismo se plantea los problemas específicos:

¿Existen diferencias en los niveles de dimensión carga laboral en los docentes de las facultades de educación de universidades estatales de la macro región centro?, ¿Existen diferencias en los niveles de dimensión clima laboral en los docentes de facultades de educación de universidades estatales de la macro región centro?, ¿Existen diferencias en los niveles de dimensión desarrollo profesional en docentes de facultades de educación de universidades estatales de la macro región centro?,

¿Existen diferencias en los niveles de dimensión bienestar en docentes de facultades de educación de universidades estatales de la macro región centro?

Ante ello se formuló el siguiente objetivo general:

Comparar las diferencias en los niveles de calidad de vida laboral en docentes de facultades de educación de universidades públicas de la macro región centro. Siendo los objetivos específicos:

Comparar las diferencias en los niveles de dimensión carga laboral en docentes de facultades de educación de universidades estatales de la macro región centro.

Contrastar las diferencias en los niveles de dimensión clima laboral en docentes de facultades de educación de universidades de universidades estatales de la macro región centro.

Contrastar las diferencias en los niveles de dimensión desarrollo profesional en docentes de facultades de educación de universidades estatales de la macro región centro. 
Comparar las diferencias en los niveles de dimensión bienestar en docentes de facultades de educación de universidades estatales en la macro región centro.

La investigación se basa sobre el estado de salud de los peruanos en las diferentes edades, géneros, zonas, urbanas, rurales, entre otros, específicamente se tiene estudios de desnutrición, sobre peso, obesidad, índice de mortalidad, etc. empero estudios de calidad de vida laboral de los docentes universitarios e incluso de Educación Básica Regular, muy pocas veces fueron tomados en cuenta como temas de estudio. La teoría de las relaciones humanas señala que es insoslayable el buen trato a los trabajadores, profesionales, etc. para un resultado efectivo en cualquiera de las áreas laborales. Los docentes universitarios para desarrollar competencias en los futuros profesionales tienen la necesidad de una preparación cada vez mayor, pero no vasta la exigencia, sino en qué medida está siendo garantizada su salubridad para responder a estas exigencias propios de una institución formadora de profesionales. Consideramos que este aspecto viene siendo dejado lado, en consecuencia, amerita una investigación.

La calidad de vida laboral implica la satisfacción de los trabajadores con su labor en las instituciones públicas o privadas y como posibles indicadores se pueden señalar como el bienestar en el aspecto físico, psíquico, emocional y social, para la consecución de estos objetivos existen algunos factores como la estabilidad laboral, clima institucional favorable, respeto a los derechos laborales, estímulos o recompensas por los resultados logrados, entre otros aspectos. Los últimos enfoques en el campo administrativo precisamente vienen priorizando las consideraciones y el buen trato a los recursos humanos, pues es una forma de garantizar el desempeño de los trabajadores. En nuestro medio en ese sentido se pueden observar que son contradictorio, se exige resultados, pero se soslaya aspectos importantes que estimule al trabajador y dinamice las actividades en las instituciones.

En el campo educativo y particularmente en las universidades los docentes, de acuerdo a algunos estudios en la mayoría de ellos arrojan como resultado la insatisfacción de los docentes universitarios por algunos factores entre ellos, el incumplimiento de los derechos laborales por parte del estado, seguido del clima institucional desfavorable generalizada en las instituciones. De otro lado, en la actualidad la educación superior universitaria se encuentra orientada al logro de las competencias profesionales y laborales a fin de asegurar a los futuros profesionales en el mercado laboral, contando como soporte la tecnología, medio que es una exigencia para los docentes que demanda la capacidad de adaptación a los cambios científicos, tecnológicos y empoderamiento de los conocimientos para su aplicación en diferentes contextos y circunstancias de manera creativa.

Por su parte, Oblitas (2014) define a la calidad de vida como: "Estado de satisfacción general de la persona, donde incluye aspectos subjetivos como objetivos. En los aspectos subjetivos se considera: expresión emocional, intimidad, seguridad que puede percibir y la productividad personal. Por otro lado, los aspectos objetivos: las relaciones que puede tener con el ambiente físico y social, teniendo en cuenta también la relación que tiene con la comunidad el bienestar material, y la salud que puede ser percibida". (p. 45)

Como manifiesta Cornejo (2014): "El mantener una calidad de vida satisfactoria o insatisfactoria induce a la adopción de un estilo de vida personal y colectiva" (p. 68). Los estilos de vida son las diferentes maneras de actuar en la vida cotidiana, el cual implica la organización del modo de vivir de las personas. Por su parte, Urzua (2008) conceptúa a la calidad de vida como: "El estado o sentimientos de bienestar derivado de la evaluación objetiva como subjetiva del grado de satisfacción de la persona en distintas dimensiones de la vida". (p. 61). 
La hipótesis general que se menciona es: No existen diferencias significativas en los niveles de calidad de vida laboral en docentes de facultades de educación de universidades públicas de la macro región centro.

Así mismo se plantea las hipótesis específicas:

a. No existen diferencias en los niveles de la dimensión carga laboral en docentes de facultades de educación de universidades estatales de la macro región centro.

b. No existen diferencias en los niveles de la dimensión clima laboral en docentes de facultades de educación de universidades estatales de la macro región centro.

c. No existen diferencias en los niveles de la dimensión desarrollo profesional en docentes de facultades de educación de universidades estatales de la macro región centro.

d. No existen diferencias en los niveles de la dimensión bienestar en docentes de facultades de educación de universidades estatales de la macro región centro.

\section{Materiales}

Para el estudio se considera el diseño descriptivo comparativo. En cuanto a la población está conformada por 360 docentes de las facultades de educación de la macro región centro, siendo la muestra de estudio de 230 docentes, a los cuales se eligen mediante el muestreo no probabilístico, de manera intencional o criterial.

Como método general se utiliza el método científico y como métodos específicos: descriptivo, deductivo y el estadístico. De tal manera para la investigación se emplea la técnica de la encuesta, la misma que nos permite recoger los respectivos datos utilizando un formulario de Google Forms el instrumento consta de 40 ítems divididos en las cuatro dimensiones.

Para aplicar el instrumento se somete a la validación de contenido en el cual participan expertos a cargo de profesionales a fin en la materia. Y para hallar la fiabilidad del instrumento calidad de vida laboral de los docentes universitarios, se considera una pequeña muestra conformada por 12 docentes, siendo el resultado de 0,922 , lo que indica que el instrumento es fiable por excelencia.

\section{Resultados}

Frecuencia de la variable calidad de vida laborable de los docentes de facultades de educación de las universidades públicas de la macro región centro

\section{Tabla 1}

Frecuencia de la variable calidad de vida laborable

\begin{tabular}{lccccccc}
\hline \multirow{2}{*}{ Niveles } & \multicolumn{2}{c}{ F. Educación. } & \multicolumn{2}{c}{ F. Educación. } & \multicolumn{2}{c}{ F. Educación. } & \\
& \multicolumn{2}{c}{ UNCP } & \multicolumn{2}{c}{ Herminio Valdizán } & \multicolumn{2}{c}{ Huancavelica } & \multirow{2}{*}{ Total } \\
\cline { 2 - 6 } & $\mathrm{ni}$ & $\%$ & $\mathrm{ni}$ & $\%$ & $\mathrm{ni}$ & $\%$ & \\
Buena $(54-80)$ & 12 & 15,00 & 8 & 8,89 & 15 & 25,00 & 35 \\
Regular $(27-53)$ & 63 & 78,75 & 79 & 87,78 & 42 & 70,00 & 184 \\
Deficiente $(0-26)$ & 5 & 6,25 & 3 & 3,33 & 3 & 5,00 & 11 \\
Total & 80 & 100,00 & 90 & 100,00 & 60 & 100,00 & 230 \\
\hline
\end{tabular}

Fuente. Archivo de la variable calidad de vida laborable 


\section{Figura 1}

Porcentaje de la variable calidad de vida laborable

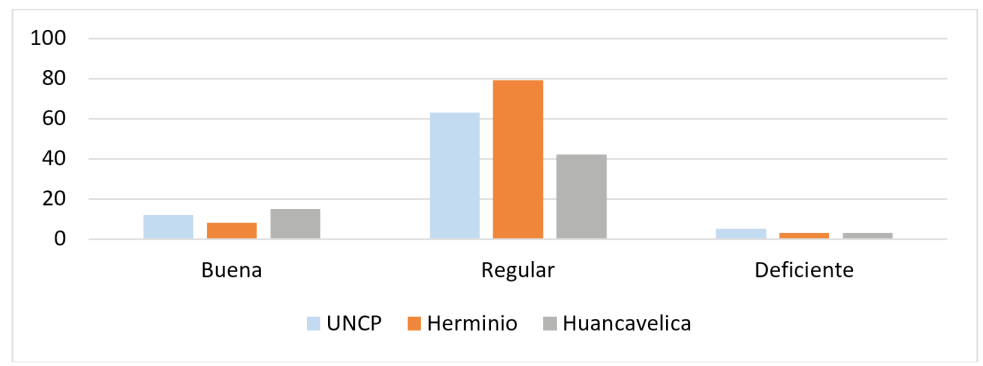

Fuente. Archivo de la variable calidad de vida laborable

A partir de los resultados de la variable calidad de vida laboral de los docentes de las facultades de Educación de las universidades públicas macro regional, se observó que los docentes de la Facultad de Educación de la UNCP el 15\% alcanzaron el nivel bueno, mientras el $78,75 \%$ se encuentran en el nivel medio o regular y el 6,25\% de docentes alcanzaron el nivel malo o deficiente. Del mismo modo los docentes de Educación de la Universidad Herminio Valdizán de Huánuco, el 8,89\% de los docentes alcanzaron el nivel bueno, el 87,78\% se lograron el nivel regular y el 3,33\% de los docentes alcanzaron el nivel deficiente.

Mientras que los docentes de Educación de la Universidad de Huancavelica, el 25\% alcanzaron el nivel bueno, el 70\% lograron el nivel medio o regular y el 5\% de los encuestados lograron ubicarse en el nivel malo o deficiente.

En relación a la contrastación de hipótesis con respecto a la variable y las dimensiones son los siguientes: como $x_{c}^{2}<x_{0,05}^{2} \quad(8,34<11,143)$, por tanto, se acepta la hipótesis nula (Ho), lo que indica la no existencia de diferencias significativas de frecuencias en la población en relación a la variable de calidad de vida laboral de los docentes de las facultades de educación de las universidades públicas de la macro región centro.

Por otro lado, como $x_{c}^{2}<x_{0,05}^{2} \quad(5,32<11,143)$, por tanto, no existen diferencias significativas de frecuencias en la población en relación a la dimensión carga laboral de los docentes de facultades de educación de las universidades estatales de la macro región centro.

Así mismo Como $x_{c}^{2}<x_{0,05}^{2} \quad(0,50<11,143)$, por consiguiente, no existen diferencias significativas de frecuencias en la población en relación a la dimensión clima laboral de los docentes de las facultades de educación de las universidades estatales de la macro región centro.

De igual manera, puesto que siendo $x_{c}^{2}<x_{0,05}^{2} \quad(1,21<11,143)$, en consecuencia, no existen diferencias significativas en la población en relación a la dimensión desarrollo profesional en los docentes de la facultad de educación de las universidades estatales de la macro región centro. 
Por último, se observa que $x_{c}^{2}<x_{0,05}^{2} \quad(6,25<11,143)$, por lo tanto, no existen diferencias significativas de frecuencias en la población en relación a la dimensión bienestar de los docentes de las facultades de educación de la universidades estatales de la macro región centro.

\section{Discusión}

Los resultados que se han obtenido producto de la aplicación del cuestionario se puede concluir, no existen diferencias significativas en los niveles de calidad de vida laboral en docentes de las facultades de educación de las universidades estatales de la macro región centro. En relación a esta variable de estudio, se observó que los docentes de la Facultad de Educación de la UNCP, el 15\% lograron el nivel bueno, el 78,75\% lograron el nivel medio o regular, mientas el $6,25 \%$ de los docentes alcanzaron el nivel malo o deficiente. Como también los docentes de Educación de la Universidad "Herminio Valdizán" de Huánuco, el $8,89 \%$ de los docentes lograron el nivel bueno, por otro lado, el 87,78\% lograron ubicarse en el nivel medio o regular, mientras que el 3,33\% de docentes se ubicaron en el nivel malo o deficiente.

Los docentes de Educación de la Universidad de Huancavelica, el 25\% alcanzaron el nivel bueno, el 70\% lograron ubicarse en el nivel medio, mientras que el 5\% de los docentes participantes lograron ubicarse en el nivel malo o deficiente en relación a la variable de estudio, donde la chi cuadrada calculada resultó menor que chi cuadrada teórica $(8,34<11,143)$, con un nivel de significancia de 0,05 .

Con respecto a los estudios relacionados al tema se tiene. Becerra, T. (2020) en la tesis cuyo título fue "estrés laboral y calidad de vida en tiempos de pandemia en docentes de escuelas con quintil 1 - Cajamarca" cuyo propósito fue relacionar la variable estrés laboral y calidad de vida en épocas de pandemia en los profesores de escuelas con quintil en una población de 175 docentes realizando el análisis estadístico de Spearman donde se obtuvo una correlación significativa e inversa $(\mathrm{Rho}=-.509)$ se concluye, existe una correlación significativa de tipo inversa.

$($ Rho $=-.509)$ entre las variables de estudio. De ello el $(46.3 \%)$ reflejan un alto nivel de estrés, donde el distrito de Jesús posee mayor incidencia de (10.9\%) y el que tiene menor incidencia es Cospán con el (2.9\%). De los evaluaos el 56\% muestra una calidad de vida baja, siendo el distrito de Jesús el que tiene mayor afectación (12.6\%) mientras que el menos afectado se encuentra Cospán(4.6\%). Asimismo, en relación a los aspectos laborales, se evidenció que los docentes entre 17 a 30 años de servicio posee un estrés laboral alto, con una proyección a una calidad de vida baja, son los que tienen ( $E L=23.4 \%$ y $C V=29.1 \%)$, en situación de docentes estables $(\mathrm{EL}=44 \%$ y $\mathrm{CV}=54,9 \%)$ y aquellos que se desempeñan en instituciones multigrados $(\mathrm{EL}=38.9 \%$ y $\mathrm{CV}=44.6 \%)$. Estos resultados coinciden con los resultados nuestros, por lo que se puede señalar que efectivamente los docentes universitarios de las facultades de educación se ubican en un nivel medio o regular con respecto a la calidad de vida laboral. En algunas dimensiones se encuentra algunas diferencias, pero no significativos que hace presumir que todos en alguna medida fueron afectados por este fenómeno de la pandemia y las formas de trabajo totalmente distinto a la labor de forma presencial.

En relación a las variables carga laboral, clima laboral, desarrollo profesional y bienestar, se puede mencionar que no hubo diferencias significativas entre los docentes de las 
facultades de educación de las universidades públicas de a macro región centro, resultados que se muestran a continuación.

Con respecto a la carga laboral de los docentes de las facultades de educación de las universidades estatales de la macro región centro, se observó que los docentes de la facultad de Educación de la UNCP, el 22,5\% se ubicaron en el nivel buena, así mismo el 73,75\% lograron ubicarse en el nivel medio o regular, mientras que el 3,75\% de los profesores se ubicaron en el nivel malo o deficiente, como también los docentes de Educación de la Universidad "Herminio Valdizán" de Huánuco, el 15,56\% alcanzaron el nivel bueno, por otro lado el $84,44 \%$ se ubicaron en el nivel regular.

Mientras que los docentes de Educación de la Universidad de Huancavelica, el 21,67\% alcanzaron el nivel bueno, el 76,67\% lograron el nivel medio o regular, mientras que el $1,66 \%$ de los encuestados se situaron en el nivel malo o deficiente ubicaron en el nivel deficiente en relación a la dimensión de estudio.

En relación al clima laboral de los docentes de las facultades de educación de las universidades estatales macro regional, se observó que los docentes encuestados de la Facultad de Educación de la UNCP, el 25\% se ubican en el nivel buena, así mismo el 67,50\% logran situarse en el nivel medio o regular y el 7,50\% de los encuestados se ubican en la categoría deficiente o malo, como también los docentes de Educación de la Universidad Herminio Valdizán de Huánuco, el 28,89\% de los encuestados se encuentran en la categoría bueno, por otro lado el $65,56 \%$ logran situarse en la categoría medio o regular, mientras que el 5,55\% de los docentes participantes se ubicaron en el nivel malo.

Mientras que los docentes de Educación de la Universidad de Huancavelica, el 26,67\% alcanzaron el nivel bueno, el 66,67\% lograron el nivel regular y el 6,66\% de los encuestados se situaron en el nivel malo en relación a la dimensión en estudio.

En relación a la dimensión desarrollo profesional de los docentes de las facultades de Educación de las universidades públicas de la macro región centro, se observó que los docentes encuestados de la Facultad de Educación de la UNCP, el 15\% se ubicaron en el nivel buena, así mismo el $80 \%$ lograron ubicarse en el nivel medio o regular y el 5\% de los encuestados se ubicaron en el nivel malo o deficiente, como también los docentes de Educación de la Universidad "Herminio Valdizán” de Huánuco, el 20\% de los participantes lograron ubicarse en el nivel bueno, por otro lado el 75,56\% lograron situarse en nivel medio o regular, mientras que el 4,44\% de los participantes lograron situarse en el nivel malo o deficiente.

Mientras que los docentes de Educación de la Universidad de Huancavelica, el 21,67\% alcanzaron el nivel bueno, el 73,33\% lograron el nivel medio o regular, mientras que el 5\% de los encuestados lograron ubicarse en el nivel malo en relación a la dimensión en estudio.

En cuanto a la dimensión bienestar de los docentes de las facultades de educación de las universidades públicas macro regional, se observó que los docentes de la Facultad de Educación de la UNCP, el 22,50\% se ubicaron en el nivel buena, así mismo el 72,50\% lograron ubicarse en el nivel medio o regular y el 5\% de los encuestados se ubicaron en el nivel malo o deficiente, como también los docentes de Educación de la Universidad "Herminio Valdizán" de Huánuco, el 15,56\% de los encuestados lograron ubicarse en el nivel buena. Por otro lado, el $83,33 \%$ de los participantes lograron situarse en el nivel medio o regular, mientras que el $1,11 \%$ de los encuestados lograron situarse en el nivel malo o deficiente.

Mientras que los docentes de Educación de la Universidad de Huancavelica, el 25\% alcanzaron el nivel bueno, el 68,33\% logran el nivel medio o regular, mientras que el $6,67 \%$ 
de los encuestados se encuentan en el nivel malo o deficiente en relación a la dimensión en estudio.

Estos resultados nos muestran claramente que no existen diferencias significativas en relación a la calidad de vida de los docentes de las diferentes universidades de la macro región centro. También a estos resultados se puede añadir que los docentes universitarios no alcanzan ubicarse en el nivel bueno de calidad de vida laboral.

\section{Conclusiones}

De acuerdo a los resultados se concluye que no existen diferencias significativas en los niveles de calidad de vida laboral en docentes de las facultades de educación de las universidades públicas de la macro región centro, puesto que chi cuadrado teórica fue mayor al chi cuadrado calculada $(11,143>8,34)$, con un nivel de significancia de 0,05 .

Se contrastó que no existen diferencias significativas en los niveles de la dimensión carga laboral de los docentes de las facultades de educación de las universidades públicas de la macro región centro, puesto que chi cuadrado teórica fue mayor que chi cuadrado calculada $(11,143>5,32)$, con un nivel de significancia de 0,05 .

Se contrastó que no existen diferencias significativas en los niveles de la dimensión clima laboral en docentes de las facultades de educación de las universidades públicas de la región centro puesto que chi cuadrado teórica fue mayor que chi cuadrado calculada $(11,143$ $>5,32$ ), con un nivel de significancia de 0,05 .

Se contrastó que no existen diferencias significativas en los niveles de la dimensión desarrollo profesional en docentes de facultades de educación de universidades públicas de la macro región centro, puesto que chi cuadrada teórica fue mayor que chi cuadrada calculada $(11,143>1,21)$, con un nivel de significancia de 0,05 .

Se concluyó que no existen diferencias significativas en los niveles de la dimensión carga laboral en docentes de las facultades de educación de universidades públicas de la macro región centro, puesto que chi cuadrada teórica fue mayor que chi cuadrada calculada $(11,143$ $>6,25)$, con un nivel de significancia de 0,05 .

\section{Referencias}

Alfonte, M. (2020) Calidad de vida laboral percibida por los profesionales de salud del Centro de Salud Vallecito I-3 y centro de salud Simón Bolívar I-3, Puno 2020 [Tesis de pregrado, Universidad Altiplano de Puno]. Repositorio institucional. Repositorio.unap.edu.pe/bitstream/handle/ UNAP/13935

Barmaimon, E. (2017). Calidad de vida. 1. 15.04.2017.- Biblioteca Virtual de Salud- S.M.U. -

Becerra, T. (2020). Estrés laboral y calidad de vida en tiempos de pandemia en docentes de escuelas con quintil 1 - Cajamarca [Tesis de pregrado, Universidad Privada Antonio Guillermo Urrelo-Cajamarca] Repositorio institucional. http://repositorio.upagu.edu.pe/bitstream/handle/ UPAGU/1388

Benach, Muntaner y Solar (2010). Empleo, trabajo y desigualdades en salud: una visión global. Icaria.

Bernal, C. (2006). Metodología de la Investigación. Para administración, economía, humanidades y ciencias sociales. Pearson Educación. 
Castilla, S., Colihuil, R., Bruneau, J. y Lagos, R (2020) Carga laboral y efectos en la calidad de vida de docentes universitarios y de enseñanza media. Revista Scielo. https://doi.org/10.1590/SciELOPreprints.

Chiavenato, I (2015). Comportamiento Organizacional. La dinámica del éxito en las organizaciones. McGraw-Hill Interamericana.

Cornejo W. (2003) Sindrome de burnout, motivación e indicadores psicosociales de calidad de vida en docentes de Ica. [Ponencia]. IX Congreso Nacional y I Congreso Internacional de Psicología. Lima.

Day, C. (2006). Pasión por Enseñar: La identidad personal y profesional del docente y sus valores. Madrid, España: Narcea

Del Águila, R. (2012) Promoción de estilos de vida saludable y prevención de enfermedades crónicas en el adulto mayor. Organización Panamericana de la Salud.

Gamarra, H. (2019). El estrés laboral y sus consecuencias en el desempeño del docente de nivel primario del distrito de Mollepata-Anta- Cusco. [Tesis de maestría, Universidad Cesar Vallejo]. Repositorio institucional. https://repositorio.ucv. Edu.pe/handle/20.500.12692/38294

Guevara, H. y Domínguez, A. (2011). Calidad de vida del docente universitario vista desde la complejidad. Revista Cubana Salud Pública, 37, 91-110. https://ww .redalyc.org/pdf/214/21419366012

Hernández, Fernández, y Baptista (2014). Metodología de la investigación. Mc Graw-Hill

Herranz, J., Reig. A., Cabrero J., Ferrer, R. y González, J. (2009). La calidad de vida, el trabajo y la salud de los profesores de la Universidad de Alicante. [ Tesis doctoral, Universidad de Alicante]. Repositorio institucional. http://rua.ua.es/ dspace/bitstream/10045/3607/1/Herranz\%20 Bellido\%2c\%20J\%c3\%bas.pdf.

Jiménez, C. (2010). Calidad de vida. Biblioteca CF+S.

Maslow, A. (1991). Una teoría de la motivación humana. En: A. Motivación y personalidad. Díaz de Santos.

Oblitas L.A. (2005) Psicología de la salud y calidad de vida. México. Edit. Thompson

Organización Mundial de la Salud (OMS). Informe mundial sobre la diabetes Geneva: OMS; 2016.

Organización Mundial de la Salud OMS (2017) Salud y derechos humanos.

Palma, S. (2005). Escala de Satisfacción Laboral (SL-SPC) Manual.

Peñaherrera, F., Cárdenas, J. y Cedillo, M. (2015) Percepción de la calidad de vida laboral de los docentes universitarios. Revista Scielo 19 no. 77 http://ve.scielo.org/scielo.php?script=sci_arttext\&pid $=\mathrm{S} 1316$

Robbins, S. \& Judge, T. (2013). Comportamiento organizacional. Edit. Pearson Educación.

Romero, M. y Laborín, F. (2016). Calidad de vida en docentes de educación pública superior. Revista Educación y Humanismo, 18(31), 205-224. http://dx.doi.org/10.17081/eduhum.18.31.1375.

Silverio, R. (2018). Calidad de vida laboral y su relación con el estrés laboral de los docentes de las I.E $\mathrm{N}^{\circ} 130$ y Antonia Moreno de Cáceres UGEL 05 SJL/EA. [Tesis de maestría, Universidad Particular Cesar Vallejo]. Repositorio institucional.https://repositorio.ucv.edu.pe/bitstream/ handle/20.500.12692

Tonon G. (2004). Calidad de vida y desgaste profesional. Una mirada al síndrome del burnout. Espacio.

Urzua A. (2008). Calidad de vida en salud. Universidad Católica del Norte 